\title{
Vorstands- und Präsidiumswahlen 2016
}

Der Präsident der DMV ruft alle DMV-Mitglieder auf, sich an der Wahl von Präsidiumsmitgliedern für die Amtszeit ab dem I. I. 2017 zu beteiligen.
Informationen über die zu besetzenden Präsidiumsplätze finden Sie auf dem diesem Heft beiliegenden Wahlzettel.

\section{Kandidatinnen und Kandidaten für die Vorstands- und Präsidiumswahlen}

\section{Schatzmeister der DMV}

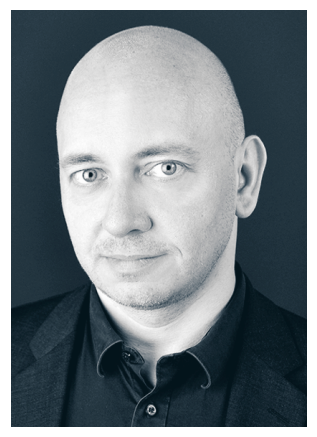

Etienne Emmrich. Geboren 1970 in Magdeburg. Studium in Magdeburg in der Fachrichtung Lehrer für Mathematik und Physik, Semester in Chemnitz und Amsterdam, Abschluss 1993 als DiplomMathematiker mit einer Arbeit zu analytischen und numerischen Aspekten hyperbolischer Erhaltungsgleichungen. Ab Oktober $1998 \mathrm{TU}$

Berlin und dort Promotion 200I über Zeitdiskretisierungsverfahren für das inkompressible Navier-StokesProblem bei Rolf D. Grigorieff (Berlin) und Lutz Tobiska (Magdeburg). Danach wissenschaftlicher Assistent bei Harry Yserentant (Berlin) und Habilitation 2007. Von Oktober 2009 bis Januar 2012 Professor für Mathematik, insbesondere Numerik, an der Universität Bielefeld. Seit Februar 2012 Professor für Mathematik, Arbeitsrichtung Differentialgleichungen an der TU Berlin.

In meiner Forschung beschäftige ich mich mit nichtlinearen Evolutionsgleichungen, die die mathematische Beschreibung zeitabhängiger Prozesse erlauben, wie die Strömung einer Flüssigkeit oder das Vibrieren einer Pipeline. Im Vordergrund steht dabei sowohl der Nachweis der Existenz verallgemeinerter Lösungen als auch die Analysis geeigneter Diskretisierungsverfahren. Ein weiterer Gegenstand meiner Forschung ist die Peridynamik, die nichtlokale Effekte in der Kontinuumsmechanik modelliert.

Neben der Forschung liegt mir die Ausbildung und Betreuung von Studentinnen und Studenten besonders am Herzen. Anderen Mathematik nahezubringen ist und bleibt für mich eine Aufgabe mit stets neuen Herausforderungen. Als Studienfachbeauftragter für den Mathematik-Service und als Geschäftsführender Direktor des Instituts für Mathematik stehe ich jedes Semester von Neuem vor der Aufgabe, die Ausbildung von rund 17000 Studienfällen jährlich bei viel zu knappen Ressourcen abzusichern. Hinzu kommt, dass Studierende von heute schlichtweg weniger Mathematik in der Schule hatten, wir bei der Hochschulausbildung aber keine Abstriche machen können. Die Gestaltung des Übergangs Schule-Hochschule und die Berücksichtigung der Bedürfnisse, der Schwächen und Stärken des einzelnen Studierenden im Massenbetrieb sehe ich als größte Herausforderung in der Lehre.

Ob uns da der auch in den Mitteilungen ausgetragene Streit zwischen den Befürwortern und Gegnern der Kompetenzorientierung so sehr weiterhilft? - Ich glaub's nicht. Dennoch ist die DMV der richtige Ort für diese und andere Diskussionen. Eine starke DMV gibt uns die Möglichkeit, in der Bildungs- und Wissenschaftspolitik Einfluss zu nehmen und zu gestalten. Mitglied der DMV bin ich seit 1994, Schatzmeister seit 2013. In meine Zeit als Schatzmeister fallen unter anderem eine Satzungsänderung, eine Beitragserhöhung und Änderung der Beitragskategorien sowie die Umstellung der Finanzbuchhaltung der DMV. Ich kandidiere für eine weitere Wahlperiode, um Kontinuität zu gewährleisten und um mich weiter für eine starke DMV zu engagieren.

\section{Herausgeber der DMV-Mitteilungen}

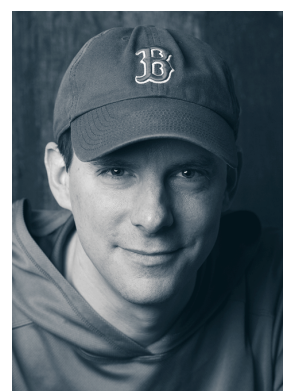

Sebastian Stiller. In Erlangen 1974 geboren. Studium der Mathematik und Philosophie in Erlangen und Leuven. Magisterarbeit bei Jens Kulenkampff über Heideggers Existenzial der Rede. Diplomarbeit über Gruppencharaktere bei Peter Plaumann. Nach all der reinen Theorie brauchte ich ein bisschen Anwendung. In der Gruppe von Rolf Möhring an der TU Berlin habe ich über Optimierung unter Unsicherheit, insbesondere robuste Optimierung, promoviert. Ich gewann ein Marie-Curie Fellowship, ging ein gutes Jahr ans MIT und forschte anschliessend wieder an der TU Berlin. Dort war ich Junior Faculty von MATHEON, Berlin Mathematical School und dem Graduiertenkolleg Methods for Discrete Structures. Im Oktober 2015 nahm ich einen Ruf zum Professor für Mathematische Optimierung an die TU Braunschweig an. 
Mein Forschungsinteresse liegt nach wie vor in der Optimierung unter Unsicherheit, v. a. für kombinatorische, mehrstufige Probleme. Mich fasziniert die Suche nach Modellen für Unsicherheit, die mächtig genug für reale Anwendungen und einfach genug für effiziente Algorithmen sind.

Mit dem populärwissenschaftlichen Buch Planet der Algorithmen (Knaus, 20I5) und daran anschliessenden Vorträgen und Interviews für Zeitung und Fernsehen bemühe ich mich um Aufklärung über das Angstthema Algorithmen.

DMV Mitteilungen: Die DMV Mitteilungen sollen das moderne Magazin bleiben, das man als Mathematiker einfach gerne liest. Sie werden unterhalten und informieren, handwerklich auf Augenhöhe zu den Wissenschaftsbeilagen überregionaler Zeitungen schreiben und diese inhaltlich insoweit übertreffen, wie es unseren Lesern entspricht. Mathematik in den Mitteilungen soll Freude machen.

Mathematik ist aber nicht nur ein Hobby. Sie ist eine der ältesten Wissenschaften. Sie war und ist Schlüssel zu neuen Technologien. Sie wirkt für Denker und Künstler als Erkenntnisideal und Inspiration. Sie ist kulturschaffend und grenzübergreifend. Mathematik ist schrecklich schön und furchtbar nützlich. Ihre technische und wirtschaftliche Bedeutung ist im Informationszeitalter enorm gewachsen. Sie war vorher schon gewaltig. Mathematik ist die Magd der Wissenschaften. Und sie besitzt das edelste Alleinstellungsmerkmal, das sich eine Wissenschaft wünschen kann: die apodiktische Gewissheit. Mathematik ist ein notwendiger Baustein des menschlichen Denkens.

Deshalb ist die DMV kein beliebiger Verein oder Interessenverband. Sie trägt Verantwortung für einen Teil dessen, was uns Menschen ausmacht. Diese Verantwortung erschöpft sich nicht in Grußworten zu Semesterbeginn und Emeritierungsfeiern. Sie ist Realpolitik.

Es geht darum, wie Mathematik in der Schule vermittelt wird, um hochschulpolitische Fragen vom Studium bis zur Förderpolitik. Es geht um technologische, wirtschaftliche und gesellschaftliche Schlüsselthemen. Es ist unsere Verantwortung, dass die Diskussion um Worthülsen wie Digitalisierung oder Big Data sinnvoll wird. Es geht um das Bild der Mathematik in der Öffentlichkeit und um ausreichende mathematische Kompetenz bei Entscheidungsträgern.

Wir Mathematiker sind uns in diesen realpolitischen Fragen nicht einig. Und das ist gut so. Aber ich bin mir einig, dass wir diese Fragen mit Sachverstand diskutieren müssen, anstatt sie Fachfremden zu überlassen. Für eine solche kontroverse, aber sachverständige Diskussion gibt es kein besseres Forum als die DMV Mitteilungen. Das öffentliche Interesse an unseren Themen ist gegeben. Unsere Diskussion wird wahrgenommen werden falls es uns gelingt, sie nicht nur kompetent sondern auch kultiviert und verständlich zu führen. Dafür will ich als Herausgeber der DMV Mitteilungen sorgen.
Herausgeber des Jahresberichtes der DMV

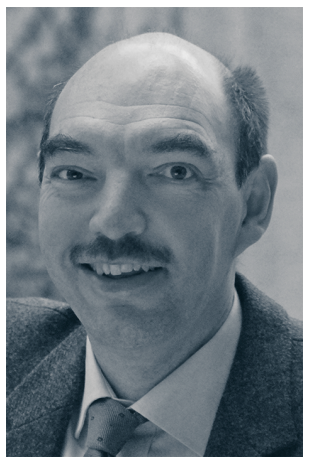

Guido Schneider. Geboren 1964 in Stuttgart, Studium der Mathematik mit Nebenfach Physik an der Universität Stuttgart, 1989 Diplom mit Schwerpunkt im Bereich stochastische Differentialgleichungen, 1992 Promotion ebenfalls an der Universität Stuttgart im Bereich nichtlineare partielle Differentialgleichungen. Von 1992-1998

an der Universität Hannover, 1997 Habilitation und Verleihung des Richard-von-Mises-Preises der GAMM. Von 1998-2002 Professor an der Universität Bayreuth, von 2002-2006 an der Universität Karlsruhe und seit August 2006 an der Universität Stuttgart. Dort von Okt. 2007 bis März 20II Prodekan und von Okt. 2014 bis Sept. 2016 Dekan der Fakultät für Mathematik und Physik. 2006-2009 Sprecher und stellvertretender Sprecher des GRK 1294 „Analysis, Simulation und Design nanotechnologischer Prozesse" an der Universität Karlsruhe, 2007-2016 PI des Excellenzclusters „SimTech“. Zur Zeit beteiligt am GRK 1838 ,Spectral Theory and Dynamics of Quantum Systems“ und am SFB I I 73 „Wellenphänomene: Analysis und Numerik“. Von 20I0-20I4 Mitherausgeber der ZAMM.

Der Schwerpunkt meiner Forschung ist die Analysis unendlichdimensionaler dynamischer Systeme. Untersucht werden meist nichtlineare partielle Differentialgleichungen auf zylindrischen Gebieten. Motiviert ist meine Forschung oft durch Wellenphänomene oder Musterbildungsprozesse. Ziele sind die Konstruktion spezieller Lösungen wie modulierter Pulse und Fronten, sowie deren Stabilitätsanalyse und insbesondere die Rechtfertigung von Amplitudengleichungen zur vereinfachten Beschreibung der Originalsysteme. Behandelt wurden u.a. die Navier-Stokes-Gleichungen, die Eulergleichungen mit freiem Rand, nichtlineare Wellengleichungen oder nichtlineare Schrödingergleichungen auch auf Quantengraphen.

Wichtig ist für mich die Verbindung der Mathematik zu den Anwendungsgebieten, sowohl in der Lehre als auch in der Forschung. Obwohl ich in meiner Forschung mathematische Sätze über das qualitative dynamische Verhalten von Lösungen partieller Differentialgleichungen beweise und Methoden aus vielen verschiedenen Bereichen der Mathematik verwende, finde ich es auch sehr spannend und inspirierend diese analytischen Resultate an der Wirklichkeit zu testen.

Ich werde versuchen, die erfolgreiche Arbeit von HansChristoph Grunau als Herausgeber der DMV Jahresberichte fortzusetzen und weiterhin verständlich und interessant über aktuelle und wichtige Entwicklungen der Mathematik auch im historischem Kontext berichten zu lassen. 
Weitere Präsidiumsplätze

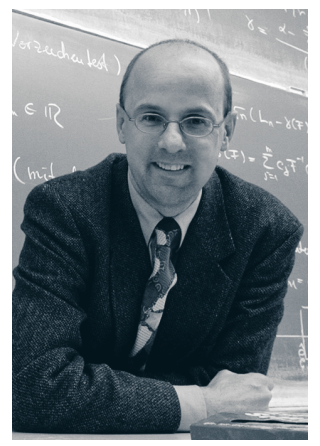

Holger Dette. Geboren 1961 in Hannover. Studium der Mathematik und Informatik an der Universität Hannover. 1987 Diplom mit Schwerpunkt Stochastik. 1989 Promotion an der Universität Hannover im Bereich der mathematischen Statistik. 19891990 wissenschaftlicher Mitarbeiter an der Universität Göttingen. 199| Assistant

Professor an der Purdue University, West-Lafayette, U.S.A. 1992 wissenschaftlicher Mitarbeiter und Habilitation an der Georg-Augustus-Universität Göttingen. 19931995 Professor für mathematische Statistik an der Technischen Universität Dresden. Seit 1995 Professor für Stochastik an der Ruhr-Universität Bochum. 2006-2008 Dekan und Prodekan der Fakultät für Mathematik an der Ruhr-Universität Bochum. Seit 2009 stellvertretender Sprecher des Sonderforschungsbereiches 823 ,Statistik nichtlinearer dynamischer Prozesse“. Seit 2012 Herausgeber des Journal of Statistical Planning and Inference. Seit 2016 Herausgeber des Journals Bernoulli und Mitglied im Fachkollegium Mathematik der Deutschen Forschungsgemeinschaft

Der Schwerpunkt meiner Forschung liegt im Bereich der Stochastik, insbesondere der mathematischen Statistik mit Beziehungen zu klassischen Gebieten der Analysis wie den speziellen Funktionen. In der mathematischen Statistik beschäftige ich mich unter anderem mit der Theorie der optimalen Versuchsplanung (Wie kann man statistische Experimente planen, um mit möglichst wenig Ressourcen maximale Informationen zu gewinnen?) und mit statistischen Tests auf Modellannahmen. In der Wahrscheinlichkeitstheorie interessiere ich mich für zufällige Matrizen und Grenzwertsätze. Weitere Arbeitsgebiete sind u. a. die Momententheorie und orthogonale Polynome.

In der Lehre ist es mir besonders wichtig, meine Begeisterung für das Fach Mathematik auch auf die Studierenden zu übertragen. Dabei möchte ich einerseits die wunderbaren Methoden und Theorien unseres Fachs vermitteln und andererseits auch erklären, dass die Mathematik die Grundlage für viele Entwicklungen in anderen Fachgebieten, insbesondere in den Naturwissenschaften, ist.

Die DMV engagiert sich vorbildlich für ein positives Bild unseres Fachs in der Politik und Gesellschaft. Ich bin gerne bereit, mich auch im Rahmen dieses Gremiums für unser Fach einzusetzen.

Bernhard Hanke. Geboren 197I, aufgewachsen in München. Studium der Mathematik und theoretischen Physik an der LMU München, der University of Cambridge und der Universität Bonn. Promotion in München im Jahr 1999. Forschungsaufenthalte und Gastprofessuren am MPI Bonn, an der University of Madison/Wisconsin, der University of Notre Dame, der Universität Göttingen, der TU Berlin und der University of Chicago. Habilitation im Jahr 2006 in Mün-

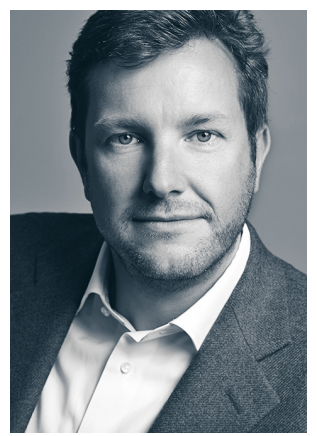
chen. Ab Sommer 2009 Professur für Differentialgeometrie und ihre Anwendungen an der TU München, im Sommer 2010 Wechsel auf den Lehrstuhl für Differentialgeometrie an der Universität Augsburg. Seit Herbst 2015 gewähltes Mitglied der Erweiterten Universitätsleitung und des Universitätsrates der Universität Augsburg. Arbeitsgebiet ist die algebraische Topologie, insbesondere in ihrer Wechselwirkung mit der Differentialgeometrie, globalen Analysis und Kombinatorik.

Mitorganisator zahlreicher wissenschaftlicher Tagungen, Engagement im Austausch von Hochschulmathematik mit Öffentlichkeit und Schule als erster Vorsitzender des Mathematisch-Physikalischen Vereins der Universität Augsburg, Nachwuchsförderung im Rahmen der Sommerakademien der Studienstiftung des deutschen Volkes und als Mitglied des Auswahlausschusses des Bundeswettbewerbs Mathematik. Seit Sommer 2015 Beauftragter der DFG für die Gauß-Vorlesungen. Koordinator des kürzlich eingerichteten DFG-Schwerpunktes „,Geometry at Infinity“, der Forschung und Nachwuchsförderung im Bereich der Differentialgeometrie, geometrischen Topologie und globalen Analysis in den kommenden sechs Jahren deutschlandweit vernetzt.

Es gibt viele Gründe, sich für unser Fach zu begeistern - jenseits von Vorlesungen, Seminaren und Konferenzen. Mathematik ist jahrtausendealtes Kulturgut, Schlüsseldisziplin der schulischen und universitären Ausbildung, Bindeglied zwischen theoretischem Denken und Anwendung, universelle Sprache der Natur- und Ingenieurwissenschaft, der Medizin, der Finanz- und Wirtschaftswissenschaft. In ihrer Doppelrolle als Natur- und Geisteswissenschaft ist sie seit jeher Quelle auch ästhetischer Inspiration. Sie ist global und verbindet Länder und Kulturen, wie ich als ein Delegierter der DFG in diesem März auf dem Next Einstein Forum in Dakar, Senegal, eindrucksvoll erleben durfte. Mathematik ist international und auBerdem durch elektronische Medien jederzeit und überall verfügbar. Warum also noch nationale Vereinigungen? Wir brauchen eine deutlich vernehmbare Stimme, die in der Öffentlichkeit den Sinn für die Aktualität, die Schönheit und die Bedeutung der Mathematik schärft. Wir brauchen ein Forum, in dem sich alle mathematisch Interessierten - Hochschulmathematiker und Studierende, Lehrer und Schüler, Vertreter aus Wissenschaft, Wirtschaft und Politik - begegnen und miteinander ins Ge- 
spräch kommen. Wir brauchen eine starke Vertretung, die unsere Interessen in den nationalen und internationalen wissenschaftlichen und politischen Diskurs einbringt. All diese Punkte sind mir wichtig, und all diese Aufgaben leistet die Deutsche Mathematikervereinigung. Daher mein Wunsch, mich in Zukunft im Präsidium der DMV zu engagieren.

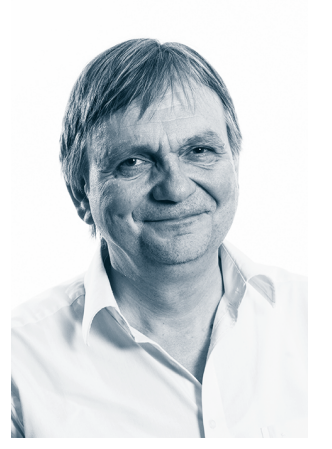

Frank Loose. Ich bin 1960 in Düsseldorf geboren und dort aufgewachsen. Studiert habe ich Mathematik und Physik zunächst an der RWTH Aachen, dann an der Universität Düsseldorf. Mein Erstes Staatsexamen, Diplom, meine Promotion und Habilitation habe ich unter Gerd Fischer in Düsseldorf abgelegt. Als Post-Doc war ich an der ETH Zürich und der Ruhr-Uni Bochum. 1995 habe ich eine Professur für komplexe Analysis an der Uni Bonn vertreten und seit Oktober 1995 bin ich an der Universität Tübingen zunächst als Hochschuldozent, später als Rat und Oberrat und heute als Akademischer Direktor tätig. 200 I wurde ich zum außerplanmäßigen Professor in Tübingen ernannt, 200I-2002 habe ich den Lehrstuhl für Topologie an der LMU München vertreten. Meine fachlichen Interessen liegen im Bereich der Funktionentheorie, Differentialgeometrie und Topologie. In den letzten Jahren habe ich mich vermehrt auch mit fachdidaktischen Themen beschäftigt.

Neben meiner akademischen Lehre habe ich mich immer schon sehr für die Öffentlichkeitsarbeit in Mathematik und ihre Außenwirkung engagiert, z. B. viele Vorträge an Schulen gehalten und überregionale Seminare für Schüler durchgeführt. Gegenwärtig mache ich in Tübingen die Vortragsreihe „Perlen der Mathematik“, die sich an Hörer aller Fakultäten richtet, auch regelmäßig Schulklassen anzieht und überraschend gut besucht ist. Seit vielen Jahren bin ich für den jährlichen „Tag der Mathematik“" in Tübingen verantwortlich, an dem etwa 250 bis 300 Schüler zu einem Wettbewerb aus weitem Umfeld an das Tübinger Institut kommen.

Ich habe einige Erfahrungen in der Gremienarbeit. Zur Zeit bin ich Studiendekan des Fachbereiches Mathematik, Vorsitzender des Prüfungsausschusses für das Lehramt Mathematik, Beauftragter des Fachbereiches für Fachdidaktik und auch uniweit präsent, etwa durch mein Amt als stellvertretender Direktor bei der neu eingerichteten Tübingen School of Education (TÜSE).

Mein besonderes Engagement gilt den Schwierigkeiten, die Studierende beim Übergang von der Schule zur Hochschule haben, nicht nur in Studiengängen der Mathematik, sondern auch in den sogenannten WiMint-Fächern, wo Mathematik ja immer noch eine große Hürde dar- stellt. Ich organisiere seit vielen Jahren die „AG Mathematik zwischen Schule und Hochschule" in Tübingen und bin Mitglied im Kernteam der Arbeitsgruppe cosh, einer Gruppe von Lehrern und Hochschullehrern in Baden-Württemberg, die zuletzt mit ihrem so bezeichneten Mindestanforderungskatalog für einige Aufmerksamkeit gesorgt hat. Im Zusammenhang mit der Ursachenerforschung von hohen Abbrecherquoten habe ich einige Projekte, die u.a. von der Deutschen Telekom Stiftung finanziert werden.

Ich finde es sehr wichtig, dass die Mathematik sich bei gesellschaftsrelevanten Themen wie z. B. der Schulbildung in Mathematik deutlich Gehör verschafft, und da ist die DMV das richtige Sprachrohr. An der Mitarbeit daran würde ich mich gerne beteiligen und kandidiere deshalb für einen der frei werdenden Präsidiumsplätze.

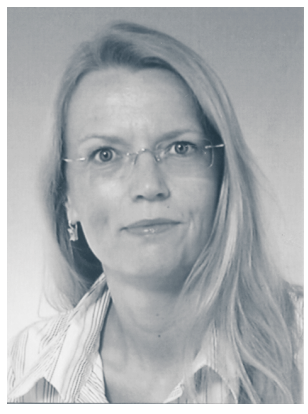

Andrea Peter-Koop. Geboren 1965 in Bielefeld, Studium des Lehramtes Primarstufe mit den Fächern Mathematik, Deutsch, Sachunterricht an der Universität Bielefeld, 1990 Erstes Staatsexamen und im Anschluss zweijähriger Forschungsaufenthalt in Melbourne, Australien. Anfang 1995 Promotion zum Dr. phil. bei Prof. Hans-Georg Steiner in Bielefeld zum Thema „Lehrerfortbildung aus international vergleichender Perspektive“. Direkt im Anschluss bis 200I wissenschaftliche Assistentin am Lehrstuhl von Prof. Peter Sorger in Münster und nach Lehrstuhlvertretungen in Gießen, Heidelberg und Oldenburg 2003 Ruf auf eine Professur für Didaktik der Mathematik an der Universität Oldenburg. Seit 20II Professorin am IDM an der Universität Bielefeld. Seit der Berufung als Professorin war ich mehrfach als Gastprofessorin im Ausland, u. a. an der University of Port Elizabeth in Südafrika und der Zhejiang International Studies University in Hangzhou, China.

In Bezug auf meine Forschung und Lehre sowie auch im Rahmen meines ehrenamtlichen Engagements liegt mir die Förderung mathematisch besonders leistungsstarker und interessierter Schülerinnen und Schüler ebenso am Herzen wie die Prävention und Therapie von Rechenschwierigkeiten. So habe ich während meiner Münsteraner Zeit damit begonnen, spezielle Angebote für mathematisch besonders interessierte Grundschulkinder zu machen und diese Aktivitäten mit der Lehrerausbildung zu verknüpfen. In Niedersachsen war ich von 20062008 zudem Jury-Mitglied beim Landeswettbewerb ,Jugend forscht - Schüler experimentieren " für den Bereich Mathematik/Informatik.

Der aktuelle Schwerpunkt meiner Forschung liegt im Bereich des Mathematiklehrens und -lernens in inklusiven Settings. Bereits seit meiner Promotion verfolge 
ich zudem das Thema Lehrerbildung auf unterschiedlichen Ebenen, seit $200 \mathrm{I}$ als Herausgeberin der Buchreihe „Mathematics Teacher Education and Development“ bei Springer sowie als Koordinatorin diesbezüglicher Topic Groups auf internationalen Tagungen, als Wissenschaftlerin im Rahmen eigener Forschungsprojekte - aktuell in einem interdisziplinären Drittmittelprojekt zur Entwicklung (mathematik-)diagnostischer Kompetenzen im Studium - und auch hochschulpolitisch wie wissenschaftlich als stellvertretende Direktorin der Bielefeld School of Education.
Aus dieser Perspektive verfolge ich auch seit einigen Jahren mit großem Interesse die Beteiligung der DMV an Diskussionen und Papieren zur Lehrerausbildung. Ich würde mich gern in der DMV engagieren und mich dort besonders für die Aus-, Fort- und Weiterbildung von Mathematiklehrer/-innen einsetzen - nicht nur aber auch angesichts der aktuellen Herausforderungen im Kontext von Inklusion und der Integration geflüchteter Kinder und Jugendlicher in unsere Schulen.

\section{DMV-Ansprechpartner/innen vor Ort}

- Aachen: Gabriele Nebe - Augsburg: Jost-Hinrich Eschenburg - Augsburg: Ralf Werner - Bamberg: Anna Susanne Steinweg - Bayreuth: Thomas Peternell a FU Berlin: Ehrhard Behrends, Günter M. Ziegler - TU Berlin: Michael Joswig, Martin Skutella - HU Berlin: Jürg Kramer - WIAS Berlin: Wolfgang König - FH Bielefeld: Claudia Cottin - U Bielefeld: Michael Röckner - Bochum: Peter Eichelsbacher, Thomas Skill - Bonn: Werner Ballmann — Braunschweig: Volker Bach —U Bremen: Dmitry Feichtner-Kozlov a JU Bremen: Dierk Schleicher - Chemnitz: Christoph Helmberg - Cottbus: Friedrich Sauvigny - Darmstadt: Stefan Ulbrich — Dresden: Andreas Thom Duisburg: Rüdiger Schultz - Düsseldorf: Kai Köhler — Erlangen: Günter Leugering $n$ Essen: Hans Niels Jahnke n Flensburg: Heinrich Lorenzen - Frankfurt am Main: Thorsten Theobald a TU Freiberg: Michael Eiermann - Freiburg: Sebastian Goette a Gießen: Thomas Bartsch - Göttingen: Thomas Schick — Greifswald: Michael Schürmann - Hagen: Winfried Hochstättler - Halle: Gernot Stroth - Hamburg: Benedikt Löwe - Hamburg-Harburg: Wolfgang Mackens - Hannover: Christine Bessenrodt - Heidelberg: Gebhard Böckle — Hildesheim: Jürgen Sander $\square$ Ho- henheim: Georg Zimmermann $\square$ Ilmenau: Carsten Trunk $\square$ Jena: Tobias Oertel-Jäger - Karlsruhe: Michael Plum — Kassel: Wolfram Koepf — Köln: Peter Littelmann — Konstanz: Claus Scheiderer - Leipzig: Hans-Bert Rademacher v Lübeck: Jürgen Prestin — Lüneburg: Silke Ruwisch — Magdeburg: Volker Kaibel - Mainz: Thorsten Raasch @ Mannheim: Alexander Schied n Marburg: Volkmar Welker — LMU München: Helmut Schwichtenberg — UniBW München: Cornelius Greither — TU München: Peter Gritzmann — Münster: Michael Joachim — HS Neubrandenburg: Gerd Teschke - Oldenburg: Daniel Grieser - Osnabrück: Holger Brenner - Paderborn: Torsten Wedhorn - Passau: Brigitte Forster-Heinlein - Passau: Niels Schwartz - Potsdam: Christian Bär n Regensburg: Guido Kings n Rostock: Roger Labahn ॥ Saarbrücken: Jörg Eschmeier - Siegen: NilsPeter Skoruppa - HfT Stuttgart: Peter Hauber —U Stuttgart: Timo Weidl - Trier: Jochen Wengenroth - Tübingen: Jürgen Hausen - HS Ulm: Günter Gramlich — Vechta: Martin Winter — Weimar: Klaus Gürlebeck n Wuppertal: Jens Hornbostel - Würzburg: Stefan Waldmann

DEUTSCHE MATHEMATIKER-VEREINIGUNG — VORSTAND UND PRÄSIDIUM Präsident Prof. Dr. Volker Bach, Institut für Analysis und Algebra, Technische Universität Braunschweig, Pockelsstraße 14, 38I06 Braunschweig, Tel. +49.53I 391 7429, vbach@tu-bs.de Vizepräsident Prof. Dr. Michael Röckner, Fakultät für Mathematik, Universität Bielefeld, Universitätsstraße 25, 33615 Bielefeld, Tel. +49.52I 106-4774 roeckner@math. uni-bielefeld.de Schatzmeister Prof. Dr. Etienne Emmrich, Institut für Mathematik, MA 5-3, Technische Universität Berlin, Straße des I7. Juni 136, 10623 Berlin, Tel. +49.3031425745, emmrich@math.tu-berlin.de Schriftführer Prof. Dr. Daniel Grieser, Universität Oldenburg, Institut für Mathathematik, Carl-von-Ossietzky-Straße 9-II, 26I29 Oldenburg, Tel. +49.44I.7983230 daniel.grieser@uni-oldenburg.de Herausgeber der Mitteilungen Prof. Dr. Michael Joswig (verantwortl.), TU Berlin, MA 6-2, Straße des 17. Juni 136, I0623 Berlin, Tel. +49.30.31475904 joswig@math.tu-berlin.de Weitere Präsidiumsmitglieder . Prof. Dr. Rudolf Grübel, Hannover , Prof. Dr. Hans-Christoph Grunau, Magdeburg (Herausgeber des Jahresberichtes der DMV) - Prof. Dr. Moritz Kaßmann, Bielefeld . Prof. Dr. Wolfram Koepf, Kassel (Verantwortlicher für www.mathematik.de) , Matthias Lippert (Lehrervertreter) - Prof. Dr. Bettina Rösken-Winter, Bochum . Prof. Dr. Katrin Wendland, Freiburg - Prof. Dr. Günter M. Ziegler, FU Berlin (Leiter Medien- und Netzwerkbüro der DMV) — Mitgliedsbeitrag 2016 (inkl. Bezug der Mitteilungen und einer gewählten Zeitschrift, Ausnahme: Studierende und Schüler beziehen nur die Mitteilungen) - regulär EUR 105,00 . bis zur Vollendung des 30. Lebensjahres EUR 50,00 . ermäßigt für Ehepaare und eingetragene Lebenspartnerschaften EUR 150,00 * ermäßigt für Studierende (Bachelor/Master/Diplom) und Schülerinnen und Schüler EUR 20,00 - Sonderbeitrag auf Antrag (z. B. bei Arbeitslosigkeit) EUR 20,00 . ermäßigt für Mitglieder der DPG/GI/GOR/GDM/MNU oder MUED EUR 90,00 EUR - ermäßigt für Reziprozitätsmitglieder (im Ausland wohnend und Vollmitglied einer Mathematischen Gesellschaft, mit der die DMV ein Reziprozitätsabkommen hat) EUR 70,00 : ermäßigt für Senioren EUR 70,00 - Zeitschriften (Jahresabo 2016 jeweils EUR 26,00), eine der folgenden Zeitschriften ist im Mitgliedsbeitrag enthalten: - Jahresbericht der DMV (Springer Verlag Heidelberg, 4 Hefte jährlich) - Journal für Mathematik-Didaktik (Springer Verlag Heidelberg, 2 Hefte jährlich) . Mathematische Semesterberichte (Springer Verlag Heidelberg, 2 Hefte jährlich) — DMV-Server dmv.mathematik.de www.mathematik.de - DOCUMENTA MATHEMATICA www.mathematik.uni-bielefeld.de/documenta/ n Medienbüro der DMV Thomas Vogt, FU Berlin (dmv.mathematik.de) - Netzwerkbüro der DMV Stephanie Schiemann, FU Berlin (dmv.mathematik.de) . Geschäftsstelle der DMV Geschäftsführerin Roswitha Jahnke (dmv.mathematik.de) • Bankverbindung Volksbank Freiburg 6955002 (BLZ 68090000 ), IBAN: DE66 6809 00000006955002 , BIC: GENODE6IFRI

Die Deutsche Mathematiker-Vereinigung e.V. ist durch den Körperschaftssteuerbescheid 2014 des Finanzamtes für Körperschaften Berlin I (Steuer-Nr. 27/640/5 I05I) vom 18.2. 2016, wegen „,Förderung von Wissenschaft und Forschung“ als wissenschaftlichen Zwecken dienend und zu den in $§ 5$ Absatz I Nr. 9 KStG bezeichneten Körperschaften gehörig anerkannt worden. Vereinseintrag: VR 380040 beim Amtsgericht Stuttgart. Umsatzsteuer-Identifikationsnummer: DE 165534138 